\title{
Field-dependent magnetic domain structure in antiferromagnetically coupled multilayers by polarized neutron scattering
}

\author{
Amitesh Paul,* Emmanuel Kentzinger, Ulrich Rücker, Daniel E. Bürgler, and Thomas Brückel \\ Institut für Festkörperforschung, Forschungszentrum Jülich GmbH, D-52425 Jülich, Germany \\ (Received 8 September 2005; revised manuscript received 10 January 2006; published 27 March 2006)
}

\begin{abstract}
We study the magnetic structure of antiferromagnetically (AF) coupled $\mathrm{Co} / \mathrm{Cu}$ multilayers (MLs) with 10 or 40 bilayers under the influence of an external field by polarized neutron scattering in specular and off-specular geometry. We observe in the spin-flip channels off-specular intensities around the $\frac{1}{2}$-order Bragg position. Based on simulations of the measured data within the distorted-wave Born approximation we find vertically correlated domains, and their domain size evolves for a sufficiently large number of bilayers along the ML stack. Small domains most likely at the top and large domains presumably at the bottom coexist within a single ML. The small domains gradually get aligned with the applied field direction around $0.5 \mathrm{kOe}$, whereas the bigger domains remain $\mathrm{AF}$ coupled up to $3.0 \mathrm{kOe}$, which is well above the apparent saturation field measured by conventional magnetometry methods. Moreover, we observe a double-peak structure at the $\frac{1}{2}$-order position for the MLs with ten as well as 40 bilayers.
\end{abstract}

DOI: 10.1103/PhysRevB.73.094441

PACS number(s): 75.70.Cn, 75.47.De, 68.35.Ct, 61.12.Ex

\section{INTRODUCTION}

Physical properties of magnetic multilayer ${ }^{1}$ (MLs) such as interlayer coupling 2,3 and giant magnetoresistance ${ }^{4-6}$ (GMR) are not only closely related to the structure of the multilayers, i.e., interface roughness and/or the evolution of the roughness along the ML stack, ${ }^{7}$ but depend also on the details of the magnetic domain structure. ${ }^{8}{ }^{8}$ Recent investigations by Langridge et al. ${ }^{8}$ and Paul et al. ${ }^{10}$ have demonstrated the usefulness of neutron techniques in determining the field dependence of the antiferromagnetically (AF) coupled domain structure. A uniformly magnetized layer with structural interface roughness will have a magnetic surface, which deviates from an ideal plane. ${ }^{11}$ This mostly vertical deviation is termed magnetic roughness. On the other hand, a nonuniform distribution of magnetization directions due to the formation of a domain structure is also a form of magnetic disorder, now predominantly in the film plane. Both types of disorder will give rise to off-specular magnetic scattering of neutrons, which can be quantified experimentally by combining specular and diffuse scattering patterns of polarized neutron. The scattering contribution from the in-plane variation due to magnetic roughness is much weaker than the normally observed variations of the scattering intensities due to structural roughness.

Recently, Borchers et al. ${ }^{12}$ have shown the dependence of GMR on the vertical correlation of domains in a coupled ML at the coercive field. The loss of GMR was attributed to the vanishing of the vertical correlation. On the other hand, vertical correlation of domains was shown to exist at the coercive field for a coupled system. ${ }^{8}$ However, though the variation of the domain size with applied field was addressed for weakly as well as strongly coupled systems, there is so far no investigation of the evolution of the domain characteristics along the growth direction of the ML. In previous work, we have observed that the strength of the AF coupling and the GMR values correlate with the structural evolution for increasing number of bilayers $(N)$ of a ML. ${ }^{7}$ In the present work, we report on the domain structure evolution in AF- coupled $\mathrm{Co} / \mathrm{Cu}$ ML stacks as observed from neutron scattering measurements. These MLs show a high degree of morphological vertical correlation and small lateral correlation of the interface roughness. ${ }^{7}$ We observe the domain structure of the Co layers to evolve from large to small vertically correlated domains along the stack. The larger ones remain AF aligned beyond the apparent saturation field, while the antiparallel alignment for the smaller breaks up at lower external fields.

\section{EXPERIMENT}

Details of the preparation and characterization of similar $\mathrm{SiO}_{2} / \mathrm{Co}(1.45 \mathrm{~nm}) /[\mathrm{Cu}(1.02 \mathrm{~nm}) / \mathrm{Co}(1.45 \mathrm{~nm})]_{N=10}$ or 40 structures as used here have been described in Ref. 7. The thickness of the $\mathrm{Cu}$ layers corresponds to the first AF coupling maximum. ${ }^{13}$ Our MLs yield GMR ratios of up to $45 \%$ as shown in Fig. 1, where we present the hysteresis loop measured by the superconducting quantum interference device (SQUID) and the GMR for the specimen with $N=40$. The samples exhibit an in-plane uniaxial magnetic anisotropy as shown by small difference in the shape of the SQUID magnetization loops measured with the external field applied parallel (red) and perpendicular (black) to the easy axis in Fig. 2: There is a sharp step in the easy-axis curve, whereas a coherent reversal takes place for the field along the hard axis. This anisotropy is induced during the growth and very likely arises from the magnetic field used for the confinement of the plasma in the magnetron sputtering unit.

Polarized neutron reflectivity (PNR) measurements are performed at the polarized neutron reflectometer with polarization analysis ${ }^{14}$ HADAS at the Jülich research reactor FRJ-2 (DIDO). The neutron wavelength is fixed at $\lambda=4.52 \AA$. The instrument is equipped with a two-dimensional (2D) detector with a special spin analyzer that covers the whole detector area and, thus, allows simultaneously measuring specular and off-specular intensities with polarization analysis. The polarization efficiencies of the polarizer and analyzer are both $95 \%$. The specimens are kept at room temperature and 


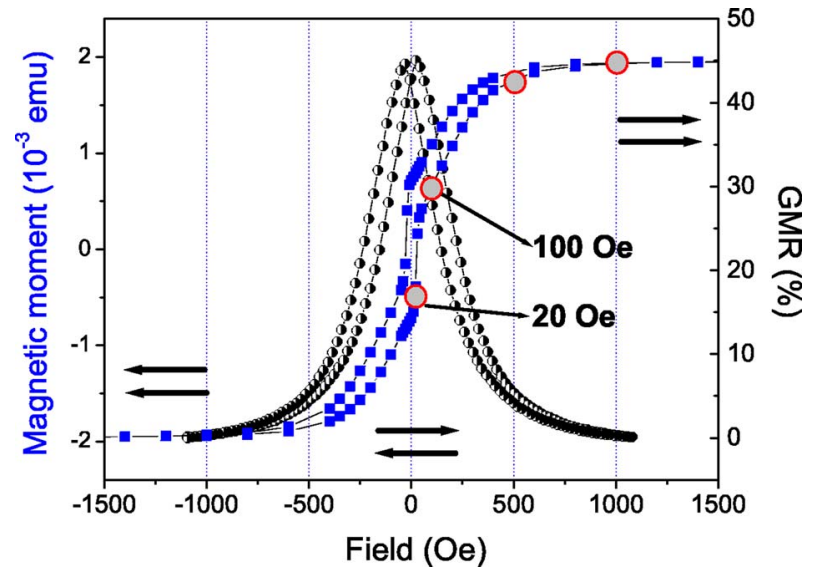

FIG. 1. (Color online) SQUID hysteresis loop [(blue) squares] and GMR curve (half-filled black circles) for a $\mathrm{SiO}_{2} /$ $\mathrm{Co}(1.45 \mathrm{~nm}) /[\mathrm{Cu}(1.02 \mathrm{~nm}) / \mathrm{Co}(1.45 \mathrm{~nm})]_{N=40}$ ML. Gray circles mark the fields of the neutron measurements shown in this paper and pairs of arrows indicate the relative magnetic alignment of neighboring Co layers.

an external field $H_{\mathrm{a}}$ of up to $4.0 \mathrm{kOe}$ has been applied. The direction of $H_{\mathrm{a}}$ is in the plane of the ML and-if not explicitly stated-perpendicular to the in-plane uniaxial easy axis of the magnetization determined from magnetization measurements like those in Fig. 2. The scattering geometry with respect to applied field direction is shown schematically in Fig. 3. We perform PNR measurements at 12 different strengths $H_{\mathrm{a}}$ of the applied field on one branch of the hysteresis loop.

Specular reflectivities in the non-spin-flip (NSF) channels $\left(R_{++}\right.$and $\left.R_{--}\right)$are due to periodicities of the structure and magnetization components collinear to $H_{\mathrm{a}}$, whereas reflectivities in the spin-flip (SF) channels $\left(R_{+-}\right.$and $\left.R_{-+}\right)$are exclusively of magnetic origin and correspond to in-plane magnetization components perpendicular to the guiding field $H_{\mathrm{a}}$. In specular geometry (angle of incidence $\alpha_{\mathrm{i}}$ equal to the exit angle $\alpha_{\mathrm{f}}$ ) the reflectivities follow from energy and in-plane momentum conservation laws. Normal wave vector transfers $Q_{\perp}=(2 \pi / \lambda)\left[\sin \left(\alpha_{\mathrm{i}}\right)+\sin \left(\alpha_{\mathrm{f}}\right)\right]$ are probed. Off-specular scat-

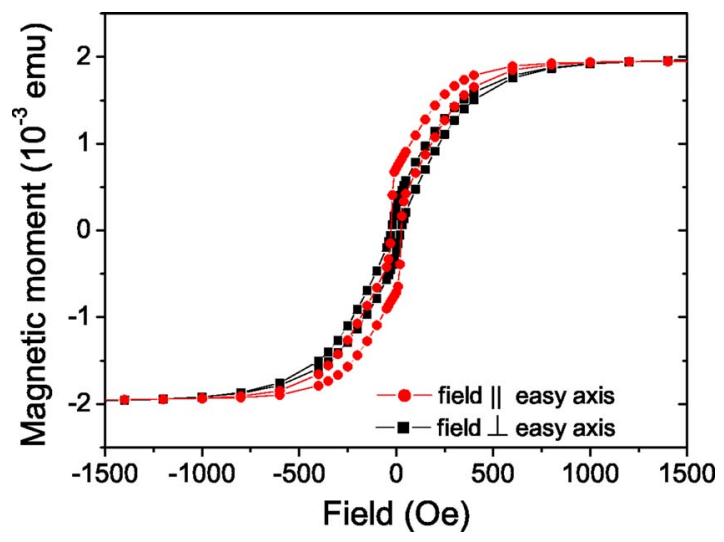

FIG. 2. (Color online) SQUID magnetization curves of the $\mathrm{SiO}_{2} / \mathrm{Co}(1.45 \mathrm{~nm}) /[\mathrm{Cu}(1.02 \mathrm{~nm}) / \mathrm{Co}(1.45 \mathrm{~nm})]_{N=40} \mathrm{ML}$ with the magnetic field applied parallel [(red) circles] and perpendicular (black squares) to the easy axis.
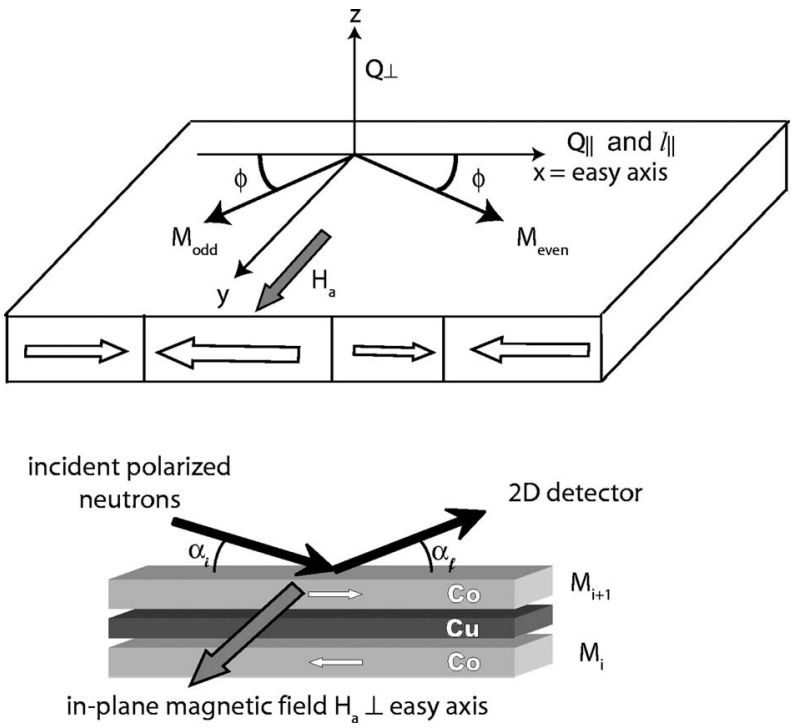

FIG. 3. Schematic drawing of the scattering geometry and its orientation with respect to the easy axis of the ML. The applied field $H_{\mathrm{a}}$ for PNR measurements is always applied perpendicular to the easy axis. $M_{\text {even (odd) }}$ denotes the magnetization direction of the Co layers with even (odd) layer number $i$ in the plane of the sample.

tering along the in-plane momentum transfer vector $Q_{\|}$ $=(2 \pi / \lambda)\left[\cos \left(\alpha_{\mathrm{f}}\right)-\cos \left(\alpha_{\mathrm{i}}\right)\right]$ arises when the in-plane translational symmetry is broken by interface roughness or magnetic domains on a length scale shorter than the in-plane projection of the neutron coherence length $l_{\|},{ }^{15,16}$ which is along $Q_{\|}$as shown in Fig. 3. For our instrument $l_{\|}$exceeds $20 \mu \mathrm{m}$, but the resolution of the $2 \mathrm{D}$ detector defines an upper limit of about $20-30 \mu \mathrm{m}$ for the resolvable lateral structure size. A lower limit of about $1 \mu \mathrm{m}$ results from the limited neutron flux at the sample position.

\section{RESULTS}

In Fig. 4 we compare the magnetization behavior as measured by the longitudinal magnetooptical Kerr effect (MOKE) and that measured by SQUID. The MOKE technique has a limited information depth due to the finite penetration depth of the laser light of about $20 \mathrm{~nm}$ (corresponding to roughly eight bilayers). Thus, for the $N=10 \mathrm{ML}$, there is very little difference between the SQUID and MOKE magnetization curves (upper panel Fig. 4). But as we grow more bilayers for $N=40$ (lower panel Fig. 4), the two curves become different. Therefore, a magnetic evolution along the stack can be suspected. However, the two techniques do not reveal significantly different saturation fields. In the following we address the magnetic domain structure and the saturation behavior by polarized neutron scattering.

Figure 5 shows the specular NSF and SF intensities as a function of incident angle $\alpha_{i}$. The intensities around the firstorder Bragg peak ( $\sim 92 \mathrm{mrad})$ are mainly due to the chemical periodicity of the ML. The $\frac{1}{2}$-order Bragg peak ( $\sim 46.5$ $\mathrm{mrad}$ ) due to the antiparallelly aligned Co layers is visible in both SF and NSF channels and for all fields except for the highest field of $4 \mathrm{kOe}$. As expected, the $\frac{1}{2}$-order Bragg inten- 


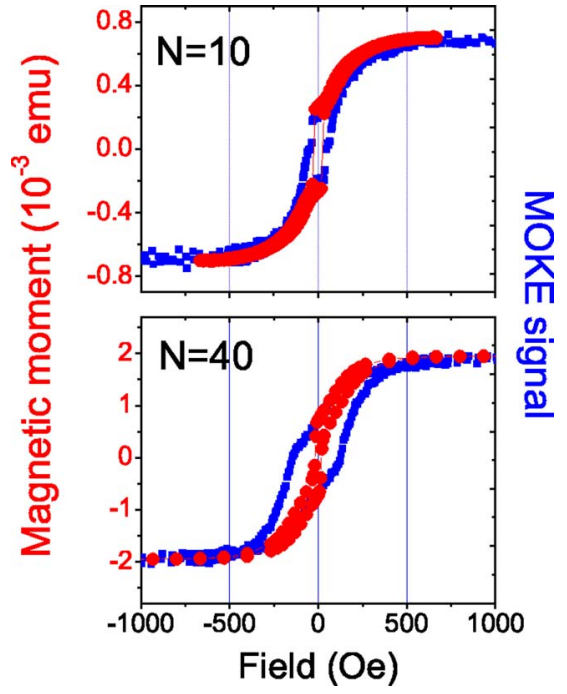

FIG. 4. (Color online) Hysteresis loops measured by longitudinal MOKE [(blue) squares] and SQUID [(red) circles] for $\mathrm{SiO}_{2} / \mathrm{Co}(1.45 \mathrm{~nm}) /[\mathrm{Cu}(1.02 \mathrm{~nm}) / \mathrm{Co}(1.45 \mathrm{~nm})]_{N}$ MLs with $N$ $=40$ and 10. The differences for $N=40$ arise from the limited information depth of MOKE and an evolution of the magnetic properties along the stack.

sities in the SF channels are more intense than in the NSF channels. No SF signal is observed when a moderate guiding field is applied parallel to the uniaxial easy axis (data not shown). Therefore, the AF-coupled layer magnetizations are collinear to the easy axis.

We fit the specular NSF reflectivities (e.g., open symbols in the left panels of Fig. 5) using the procedures described in Ref. 17: We only consider deviations from the purely collin- ear single-domain configurations and take into account the nonideal polarization efficiencies. The Co layers $(i=1, \ldots, 10$ or 40) are described by their mean magnetization amplitude $\left\langle M_{\mathrm{i}}\right\rangle$ and their angular deviation from the easy axis, which we describe by a single parameter $\phi$. In remanence, the AF coupling forces the magnetizations of adjacent Co layers to collinear, antiparallel alignment, e.g., all magnetizations $M_{\mathrm{i}}$ with even $i$ point along the $+x$ direction and all $M_{\mathrm{i}}$ with odd $i$ along the $-x$ direction. As we increase the field applied in the $-y$ direction even and odd layer magnetizations rotate symmetrically by $\phi$ toward the field (Fig. 3). In saturation, $\phi=90^{\circ}$. The fitted $\left\langle M_{\mathrm{i}}\right\rangle$ values do not show significant variations. Already around 500 Oe we find large magnetization components with parallel alignment $\left(\phi \approx 80^{\circ}\right)$. The SF intensities near $46 \mathrm{mrad}$ for $H_{\mathrm{a}}=500$ Oe are larger than the NSF intensities due to diffuse scattering and indicates the presence of lateral inhomogeneities caused by domains. This is best seen in the SF intensity maps shown in Fig. 6 for different $H_{\mathrm{a}}$. The $\frac{1}{2}$-order Bragg peak at $\sim 46.5 \mathrm{mrad}$ is broad along $Q_{\|}$as well as along $Q_{\perp}$. Its intensity rapidly shrinks with distance from the specular line and also decreases with increasing $H_{\mathrm{a}}$. Although all four channels are fitted within the single domain approximation, we note in Fig. 5 that the widths of the $\frac{1}{2}$-order Bragg peak of the SF intensities in Fig. 5 could not be reproduced because these peaks are diffuse in nature. We also like to mention, that our fits yield a structural interface rms roughness $\sigma \approx 0.6 \mathrm{~nm}$, which is in good agreement with the values from our previous $\mathrm{x}$-ray reflectivity measurements. ${ }^{7}$

The presence of this broad $\frac{1}{2}$-order Bragg peak reflects magnetic inhomogeneities along the sample plane on a length scale smaller than $l_{\|}$which are also vertically correlated. These correlations are due to columnar domains in the

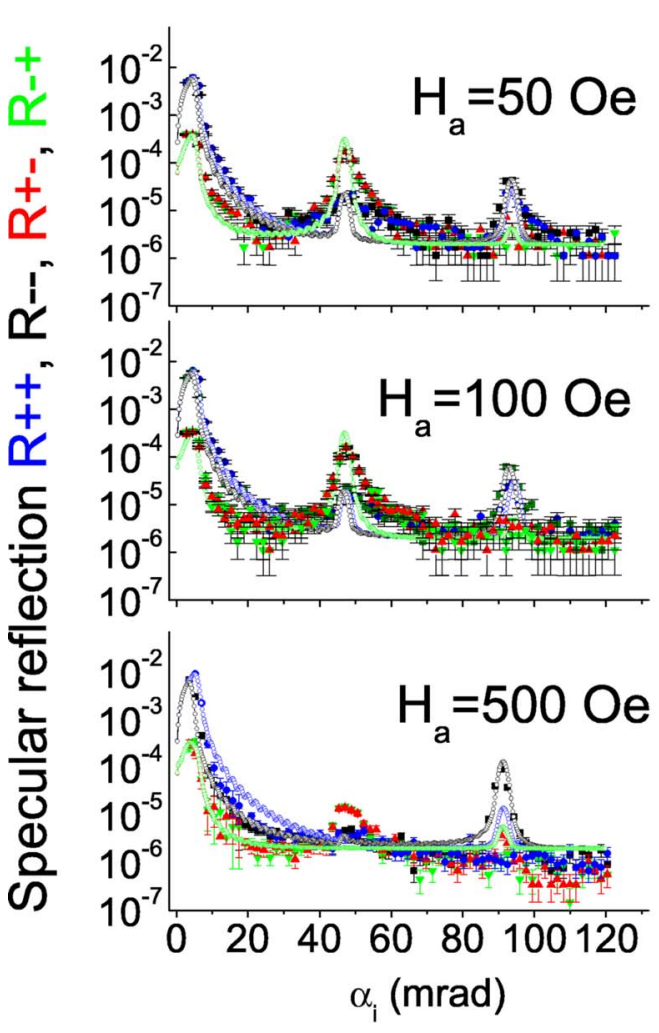

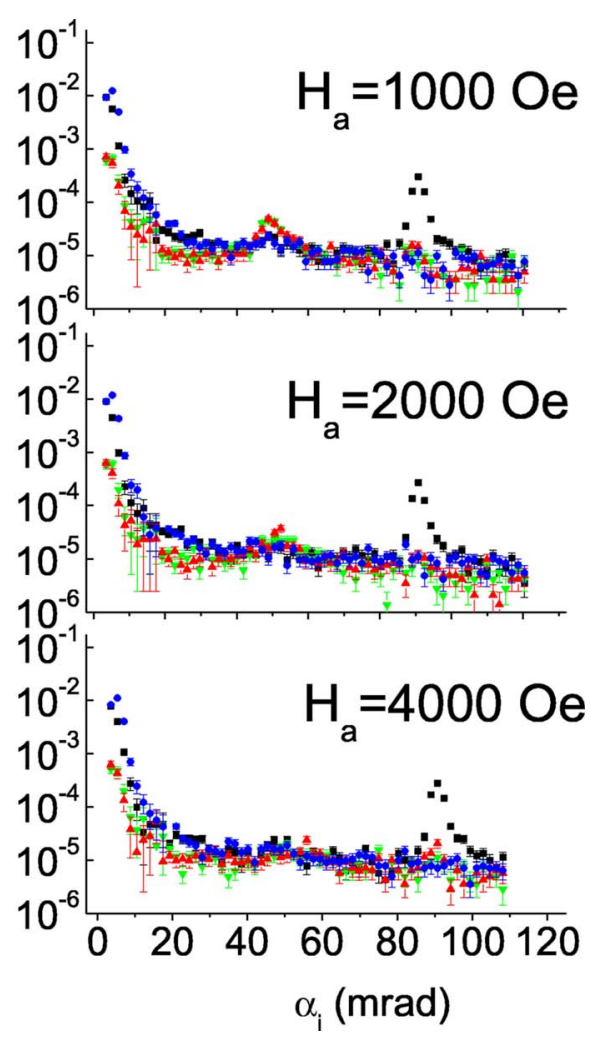

FIG. 5. (Color online) Measured NSF $\left[R_{++}\right.$(blue) circles and $R_{--}$black squares] and $\mathrm{SF}$ $\left[R_{+-}\right.$(red) triangles and $R_{-+}$ (green) downtriangles] reflectivity curves of a $\mathrm{SiO}_{2} / \mathrm{Co}(1.45 \mathrm{~nm}) /$ $[\mathrm{Cu}(1.02 \mathrm{~nm}) / \mathrm{Co}(1.45 \mathrm{~nm})]_{N=40}$ ML at different applied fields $H_{\mathrm{a}}$ as indicated. The open symbols in the left panels represent fitted curves for all the four channels of polarization. 


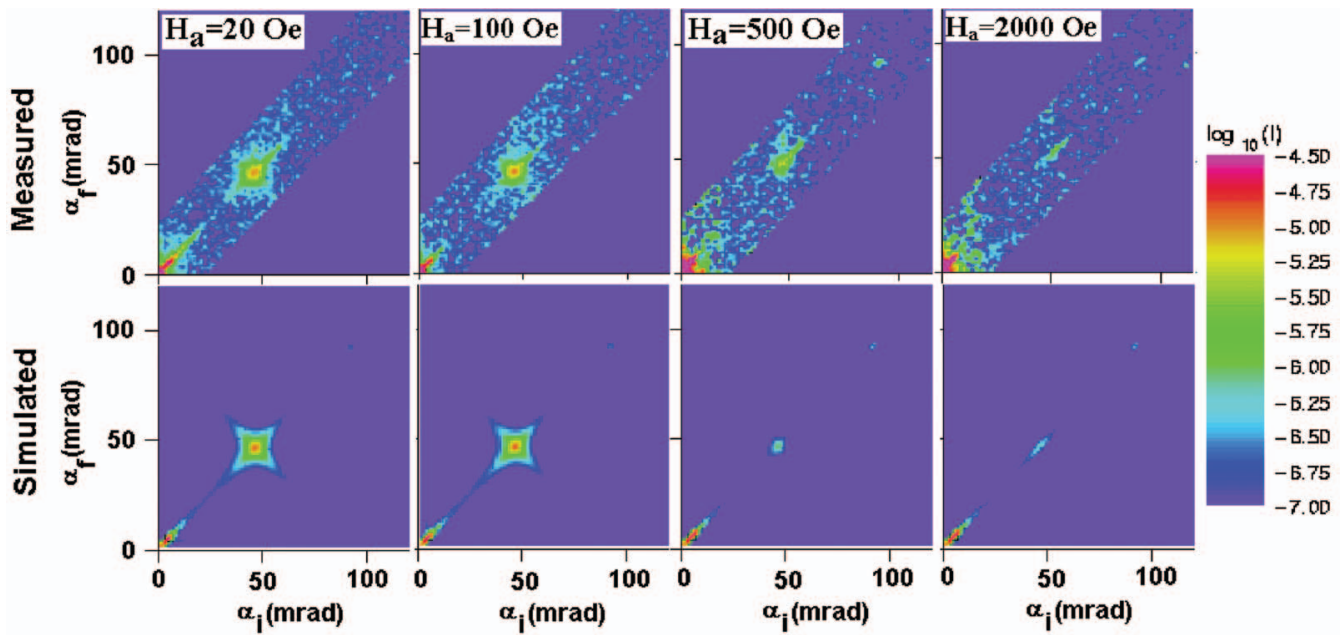

FIG. 6. (Color) Measured (upper panels) and simulated (lower panels) SF intensity maps $\left(I_{-+}\right)$of a $\mathrm{SiO}{ }_{2} / \mathrm{Co}(1.45 \mathrm{~nm}) /$ $[\mathrm{Cu}(1.02 \mathrm{~nm}) / \mathrm{Co}(1.45 \mathrm{~nm})]_{N=40} \mathrm{ML}$ at different applied fields $H_{\mathrm{a}}$ as indicated.

AF-coupled Co layers. As SF intensities arise from the magnetization components perpendicular to $H_{\mathrm{a}}$, we find that some columnar domains maintain the AF-coupled state and their magnetizations remain along the easy axis (i.e., perpendicular to $H_{\mathrm{a}}$ ). This state is energetically stable as long as the interlayer coupling prevails over the Zeeman energy.

We estimate the lateral size $\xi$ of the vertically correlated domains by simulations of the intensity maps under the distorted-wave Born approximation (DWBA) as has been described in Ref. 18. We use the fitted values of the angle $\phi$ as determined from NSF reflectivities and consider for the simulations a Gaussian fluctuation of these angles, which is denoted as $\left\langle\Delta \phi_{\mathrm{i}}\right\rangle$. It is estimated to be approximately $\pm 15^{\circ}$ for all fields and describes the fluctuation of the angle from domain to domain. These fluctuations are the origin of the enhanced intensities around the $\frac{1}{2}$-order peak in the SF as well as in the NSF channels as shown in Ref. 10. The simulations are done simultaneously for the NSF and SF specular and off-specular intensities. The results are plotted in Fig. 7. The domain size $\xi$ increases from $0.5 \mu \mathrm{m}$ at 20 Oe to $\sim 1.5 \mu \mathrm{m}$ around $1.0 \mathrm{kOe}$. These domain sizes are comparable to earlier observations. ${ }^{8,12}$ Saturation $\left(\phi=90^{\circ}\right)$ is reached for $H_{\mathrm{a}}>500 \mathrm{Oe}$. Beyond $1.5 \mathrm{kOe}$, the domain sizes grow rapidly and become larger than $l_{\|} \approx 20 \mu \mathrm{m}$. Thus, the length scale of their lateral spread can no longer be determined quantitatively (dashed line in Fig. 7). Alternatively, an estimation of the domain sizes can be obtained from the widths of the transverse cuts of the SF diffuse scattering intensities, which are inversely proportional to the in-plane magnetic correlation length or to the average domain size.,12 We extract the diffuse intensities around the $\frac{1}{2}$-order peak along $Q_{\|}$from a transverse band with a spread of a few mrad along $Q_{\perp}$. Lorentzian fits of the data are shown in Fig. 8. The estimated domain sizes (triangles) are also plotted in Fig. 7 along with those obtained from simulations under the DWBA (squares). The two different ways of estimating the domain sizes result in similar values. However, we note that from the intensities within the transverse cuts along $Q_{\|}$alone it is not possible to determine a realistic state of the magne- tization precisely, as the information regarding domain-todomain fluctuations of the magnetization can only be extracted from a simulation of the full intensity map (Fig. 6).

The in-plane diffuse scattering intensity around the $\frac{1}{2}$-order peak beyond apparent saturation is caused by different domain sizes in the ML stack, which yield different interlayer coupling strengths. Based on the comparison of MOKE and SQUID in Fig. 4 it is very likely that the different domain sizes occur at different depths of the ML stack. Note that the presence of domains beyond the apparent saturation is not revealed by the specular NSF scattering. In spite of a collinear alignment at 500 Oe deduced from the fits to the NSF reflectivities, the diffuse scattering intensities in the SF channels persists much beyond the apparent saturation field. In Fig. 9 we compare the longitudinal MOKE signal

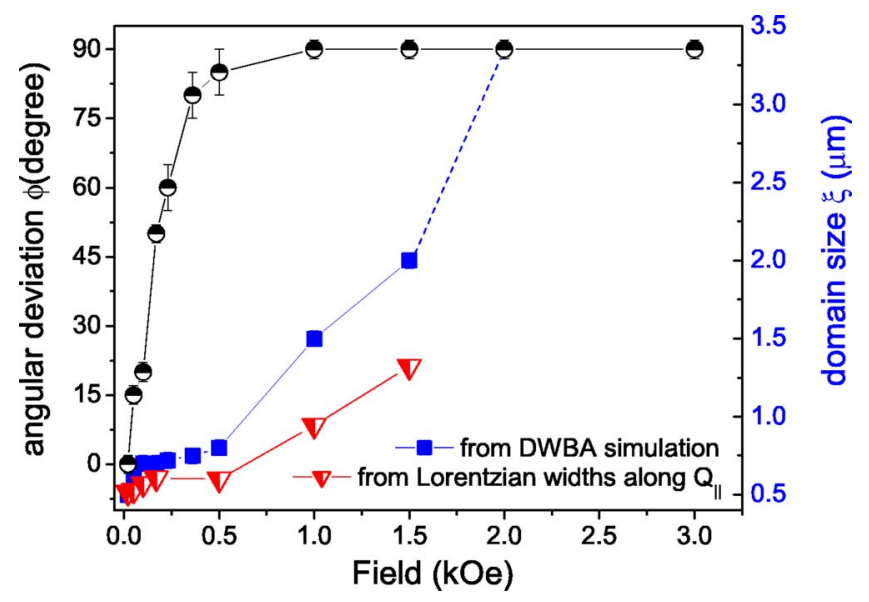

FIG. 7. (Color online) Domain size $\xi$ from DWBA simulatuions [(blue) squares, right ordinate] and from Lorentzian widths along $Q_{\|}$ [(red) half-filled down triangles, right ordinate] and angular deviation $\phi$ (black half-filled circles, left ordinate) of the layer magnetizations with respect to the easy axis as a function of the applied field strength. The data are derived from the simulations and fits to the diffuse and specular scattering from the ML with $N=40$. The lines are guides to the eyes. 


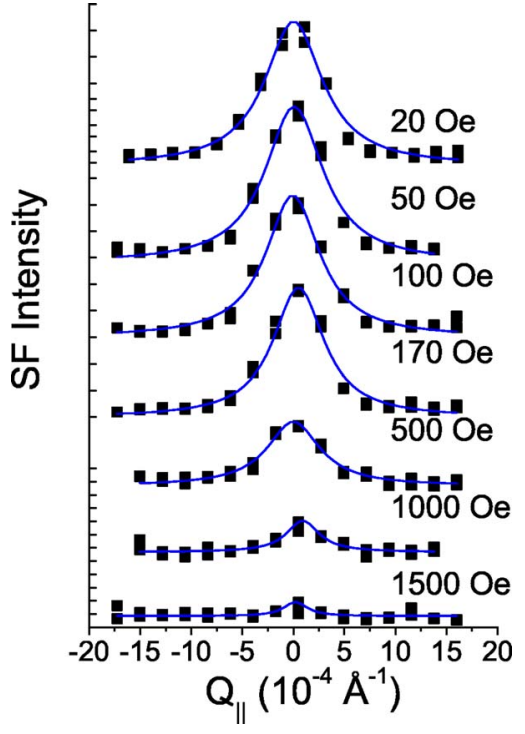

FIG. 8. (Color online) Lorentzian fits [(blue) solid lines] to the total SF intensities $\left(R_{+-}+R_{-+}\right)$along $Q_{\|}$in the vicinity of the $\frac{1}{2}$-order Bragg peak of the $N=40 \mathrm{ML}$ for different $H_{\mathrm{a}}$. The width of the Lorentzian is related to the domain size.

and SQUID response to the magnetization component parallel to $H_{\mathrm{a}}$ obtained from the neutron measurements. The latter is proportional to $\sin (\phi)$; see Fig. 3. Conventional magnetometry and PNR data in the NSF channels well agee on the apparent saturation field. The reason is that a small deviation of the layer magnetizations $(\Delta \phi)$ from the collinear alignment along the applied field direction $\left(\phi=90^{\circ}\right)$ does not make any significant difference in the magnitude of the parallel component compared to the perfect alignment: $M_{\|} / M_{S}$ $\propto \sin (90-\Delta \Phi) \approx 1-(\Delta \Phi)^{2} / 2$. In contrast, the SF diffuse scattering at the $\frac{1}{2}$-order peak is sensitive to the components perpendicular to $H_{\mathrm{a}}, M_{\perp} / M_{S} \propto \cos (90-\Delta \Phi) \approx \Delta \Phi$. Therefore, the SF diffuse scattering measurements in specular and off-specular geometry reveal the extent of the true saturation field beyond the apparent one. Without the SF diffuse scattering intensities it would not be possible to track the exis-

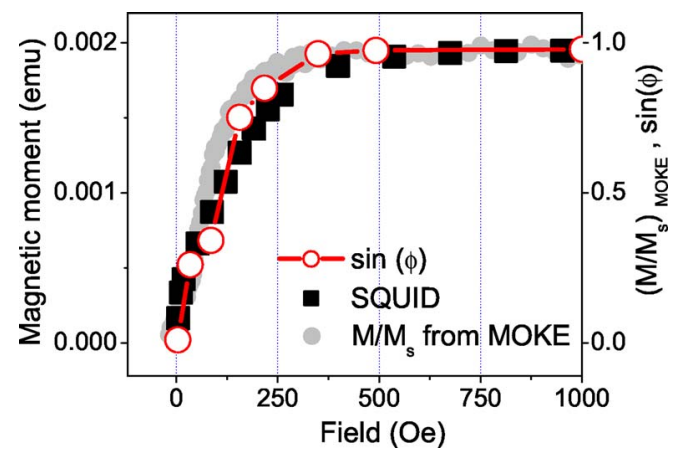

FIG. 9. (Color online) Magnetization component parallel to the direction of the applied field for the $N=40 \mathrm{ML}$ as obtained from MOKE (gray circles, right ordinate), SQUID (black squares, left ordinate), and from the fits to the NSF reflectivities given by $\sin (\phi)$ [(red) open circles, right ordinate]. Left and right ordinates are adjusted at zero magnetization and at saturation.
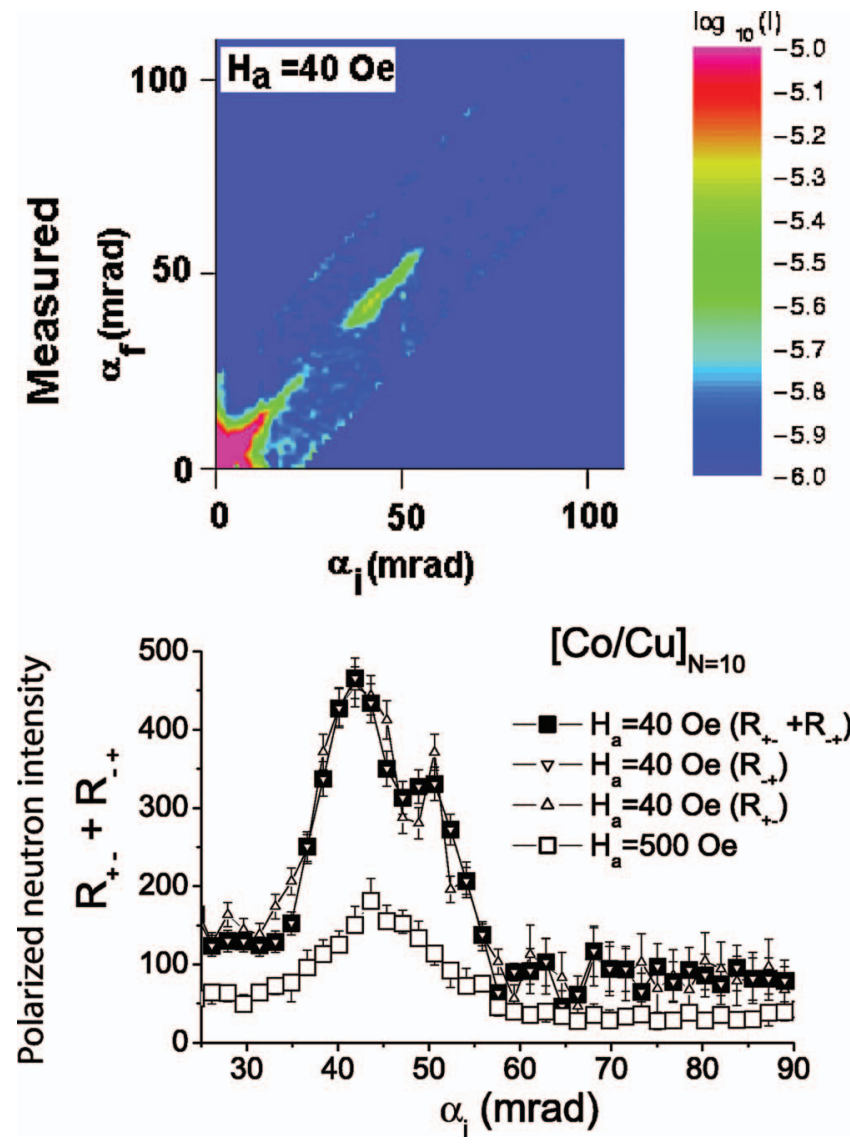

FIG. 10. (Color) Experimental SF intensity map $\left(I_{-+}\right)$of a $\mathrm{SiO}_{2} / \mathrm{Co}(1.45 \mathrm{~nm}) /[\mathrm{Cu}(1.02 \mathrm{~nm}) / \mathrm{Co}(1.45 \mathrm{~nm})]_{N=10}$ ML at $H_{\mathrm{a}}$ $=40$ Oe. The lower panel shows the total SF reflectivity $\left(R_{+-}\right.$ $\left.+R_{-+}\right)$along with the reflectivity in the individual channels $\left(R_{+-}\right.$ and $R_{-+}$) extracted along the specular line at $H_{\mathrm{a}}=40$ and $500 \mathrm{Oe}$. The shoulder from the unexpected peak is more prominent in the $R_{-+}$channel.

tence of AF-coupled domains for fields higher than the apparent saturation field. Even more, we could extract from the simulations under the DWBA small-scale domain-to-domain fluctuations which are only observable along the $Q_{\|}$direction. In this way we are able to obtain a much more detailed picture about the realistic magnetic state. ${ }^{19}$ Thus, even though the large proportion of the sample's magnetic domains are already ferromagnetically aligned by the external field, neutron scattering can reveal very small nonsaturated proportions in which AF coupling is still present. The SQUID data in which the total moment is measured, often may be affected by the instrumental background. Intensities owing to the AF coupling appeared at a different $\mathrm{Q}$ value than that due to the FM coupling which allows background free measurements of the remaining AF-coupled regions.

In order to verify the depth dependence of the domain sizes we present in Fig. 10 PNR data of another ML which is grown with the same individual layer thicknesses, but only $N=10$ bilayers: $\mathrm{SiO}_{2} / \mathrm{Co}(1.45 \mathrm{~nm}) /[\mathrm{Cu}(1.02 \mathrm{~nm}) /$ $\operatorname{Co}(1.45 \mathrm{~nm})]_{N=10}$. The upper part of Fig. 10 shows the SF intensity map measured at $H_{\mathrm{a}}=40$ Oe, i.e., close to the remanent state. The off-specular intensity of the $\frac{1}{2}$-order peak 
due to vertically correlated domains is restricted to much lower $Q_{\|}$values (compare to the panels $H_{\mathrm{a}}=20$ and 100 Oe in Fig. 6). Therefore, we suspect, all correlated domains are for $N=10$ much larger than for $N=40$. The lower part of Fig. 10 shows the total SF reflectivity $\left(R_{+-}+R_{-+}\right)$along $Q_{\perp}$ at $H_{\mathrm{a}}=40$ and 500 Oe. Surprisingly, we observe at 40 Oe two peaks with similar full width at half maximum (FWHM) of about $10 \mathrm{mrad}$. At 500 Oe the two peaks are merged into one with a FWHM of $20 \mathrm{mrad}$. This does not indicate a complete saturation because a weak shoulder is still visible. The disappearance of the $\frac{1}{2}$-order intensity already at about $500 \mathrm{Oe}$ and larger fields (data not shown) is believed to be due to the lack of sufficient SF scattering intensity. For the $N=10 \mathrm{ML}$ the intensity must be expected to be even weaker than the overall weak signal from ML with $N=40$, because the amount of magnetic material is four times less. Thus, we believe that for $N=10 \mathrm{ML}$ (unlike $N=40 \mathrm{ML}$ ) we could not observe the true saturation field by neutron scattering measurements. Even for $N=40 \mathrm{ML}$ we could observe the SF intensities above the apparent saturation fields only by allowing a measuring time which is five times longer than for fields below 500 Oe. The diffuse intensities of the $N=10 \mathrm{ML}$ structure were visible when the measuring time was increased by another factor 10 (i.e., about 56 hours per field value). Thus, the limited intensity is presently a severe restriction because even longer measuring times are not practical. Only the advent of brighter neutron sources, as for instance spallation sources, will allow one to extend experiments to thinner magnetic layers.

We now return to the $N=40 \mathrm{ML}$ structure to check the presence of a double-peak structure near the $\frac{1}{2}$-order Bragg position. A similar analysis of the $\frac{1}{2}$-order peaks as presented in Fig. 10 for the $N=10 \mathrm{ML}$ is shown in Fig. 11 for $N=40$. These data also reveal apart from the expected peak at $\sim 46.5 \mathrm{mrad}$ an unexpected adjacent peak at $\sim 52.0 \mathrm{mrad}$ with much lower intensity. The weaker peak at $\sim 52.0 \mathrm{mrad}$ seems to be independent of $H_{\mathrm{a}}$ up to $2 \mathrm{kOe}$, and it vanishes only above $3 \mathrm{kOe}$. Persistent existence of the unexpected peak has been observed over various fields at different periods of reactor operations for both the MLs. In the data shown in the Fig. 11, it becomes clearly visible at a higher value of $Q_{\perp}$ than the expected peak which has already disappeared below $2 \mathrm{kOe}$. This can be also seen in the SF maps of Figs. 6 and 10 where the extended tail of the $\frac{1}{2}$-order peak is only along the higher- $Q_{\perp}$ side and is much above the background intensities. Therefore, we fit it by a Gaussian in the top left panel of Fig. 11. The fitted Gaussian is subtracted as a background in all other panels before fitting the remaining sharper peak by a Gaussian, too. The FWHM of the subtracted Gaussian is $10 \mathrm{mrad}$ and is, thus, similar to the width of the peak in Fig. 10 at 40 Oe. Obviously, the sharper peak gradually drops in intensity and disappears for $H_{\mathrm{a}}$ $>1.5 \mathrm{kOe}$, while the FWHM increases from 50 to $1500 \mathrm{Oe}$ by $\sim 5 \mathrm{mrad}$. This increased widths is due to some loss of vertical correlation of the domains along the ML stack at roughly the same field where the diffuse intensities along $Q_{\|}$ also disappear. We find no corresponding changes of the NSF peak widths, the lack of which confirms that the observed variations are due to the evolution of the magnetic structure.

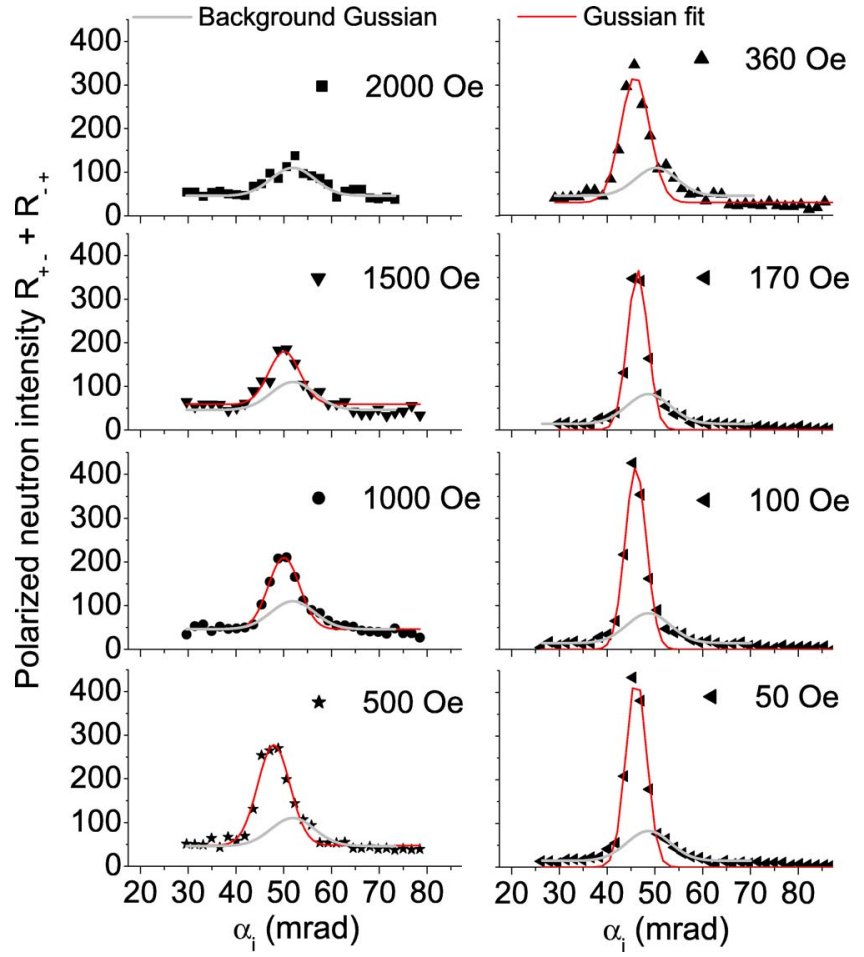

FIG. 11. (Color online) Total specular SF reflectivity $\left(R_{+-}\right.$ $\left.+R_{-+}\right)$around the $\frac{1}{2}$-order Bragg peak of the $N=40 \mathrm{ML}$ for different $H_{\mathrm{a}}$. The broad peak with low intensity at $2 \mathrm{kOe}$ (gray lines) is fitted and subtracted from all other curves prior to fitting the remaining sharper peak (red lines), see text.

\section{DISCUSSION}

The double-peak structure at the $\frac{1}{2}$-order Bragg position cannot be attributed to two different structural periodicities at different vertical levels of the stack or at two macroscopically different lateral sample positions of a single ML, because (i) no such double-peak structure is visible in the NSF channels at the first-order Bragg peak position and (ii) the unexpected peak can be suppressed by a strong magnetic field. Furthermore, the double peaks appear for two different MLs with different $N$ prepared in different deposition runs. The field where the second, unexpected peak disappears is above $3.0 \mathrm{kOe}$ for $N=40$. For $N=10$ we expect the disappearance only above $0.5 \mathrm{kOe}$, but it could not be observed owing to the weak magnetic scattering intensity above $0.5 \mathrm{kOe}$ from the $N=10 \mathrm{ML}$. However, a correlation with field is seen for the sharper expected peaks, that vanishes first: above $1.5 \mathrm{kOe}$ for $N=40$ and $0.5 \mathrm{kOe}$ for $N=10$. In earlier studies of $\mathrm{Co} / \mathrm{Cu}$ MLs grown by similar sputtering techniques, ${ }^{8,12}$ the double peak structure was possibly not resolved because (i) $N \geqslant 20$, (ii) the applied fields were limited (200-250 Oe) and did not allow one to observe the different field behavior of the two peaks, and (iii) the $\mathrm{Cu}$ thicknesses were larger corresponding to the second coupling maximum or beyond. At present we cannot explain the origin of the magnetically induced unexpected peak. Further investigations are needed. Whatever the explanation will be, we can, however, state that the unexpected peak arises from vertically correlated large domains $(\xi>l \|)$ with smaller effective magnetic periodicity. 
The expected $\frac{1}{2}$-order Bragg peak indicates (i) the formation of vertically correlated, columnar domains. Their size evolves with (ii) increasing applied field and also with (iii) the number of bilayers along the stack. In our previous work ${ }^{7}$ we have seen that the interface roughness decreases from a value comparable to the $\mathrm{Cu}$ thickness at the bottom of the ML to a significantly lower value in the top layers. In the regime, where the root-mean-square roughness is smaller than the $\mathrm{Cu}$ layer thickness, the $\mathrm{Cu}$ layers are continuous. Thus, we expect the formation of vertically correlated domains. In the other regime, domain wall pinning by the structural defects (interface roughness and/or interdiffusion extends up to few bilayers, ${ }^{7}$ e.g., $N=5$ ) leads to less correlation. With increasing $H_{\mathrm{a}}$ the smaller and weaker AF-coupled domains become ferromagnetically aligned, whereas the larger and thus stronger AF-coupled domains still remain in the antiparallel state. The SF intensity maps in Fig. 6 show the coexistence of diffuse scattering intensity arising from small as well as large domains. When the smaller domains are ferromagnetically aligned at larger fields, the peak intensity is solely due to the presence of larger domains. The small domains have a size $\xi<1.5 \mu \mathrm{m}$ as estimated from the simulations and fits (Figs. 6 and 8) and for the large domains $\xi$ clearly exceeds $1.5 \mu \mathrm{m}$. Therefore, this picture explains the increase of the average domain size with $H_{\mathrm{a}}$ (Fig. 7), and it is also consistent with our earlier findings for similar MLs, where the AF coupling fraction and the GMR ratios were found to apparently saturate beyond ten bilayers. ${ }^{7}$

Langridge et al. ${ }^{8}$ reported vertically correlated domains at the coercive field. However, these measurements were limited beyond $H_{\mathrm{a}}=200$ Oe to the experimental background. A set of domains with partly parallel and partly antiparallel alignment is likely to coexist. ${ }^{20}$ Borchers et al. ${ }^{12}$ reported such a coexistence, but it could not be restored after demagnetization. In contrast, our results show the presence of a stable remanent state with coexistent small and large domains, because we measure our samples after numerous field cycles. Our results resolve a further contradiction of previous results: The decrease in GMR was related to a loss of vertical correlation of antiparallel domains at the coercive fields in Ref. 12, whereas highly correlated domains are found to be present at the coercive field in Ref. 8. Our data show strong vertical correlation of small AF-coupled domains for the low-field range $\left(H_{\mathrm{a}} \sim 20-500 \mathrm{Oe}\right)$. At the coercive field these domains get aligned with the external field and cause the GMR effect. However, the larger domains stay AF coupled and correlated for much higher fields beyond apparent saturation.
As the system is strongly coupled, it is difficult to estimate the position of the large and small domains along the stack. The saturation fields for isolated multilayers (i.e., bottom ten bilayers and the rest of the $N=40 \mathrm{ML}$ ) are expected to be quite different from the situation when the two parts are joined together. However, it seems reasonable to assume that the larger domains, which are observed for $N=10 \mathrm{ML}$, would also form in the same way for the first roughly ten bilayers of an $N=40 \mathrm{ML}$ such that the bottom part of the $N=40 \mathrm{ML}$ will have the larger domains which gradually become smaller with increasing $N>10$. Atomic force microscopy pictures of the completed MLs (not shown here) reveal clustering of individual grains $(\approx 70 \mathrm{~nm})$ for the $N=40 \mathrm{ML}$, whereas for $N=20$ ML these grains are uniformly distributed over the surface. This structural difference cannot be seen in the neutron data because (i) of the limited lateral resolution $(\geqslant 1 \mu \mathrm{m})$ of our experimental setup and (ii) the clustering possibly only affects the surface region and do not produce enough contrast for neutrons. However, the different diffuse scattering intensity distributions at the $\frac{1}{2}$-order Bragg peak and at the structural first-order Bragg peak (e.g., in Fig. 6) immediately tell us that the lateral domain sizes and their evolution with $N$ are not related to structural features of the same lateral extent. For instance, we can directly exclude that the domain size is given by the grain size with the grain boundaries acting as pinning sites for the domain walls.

\section{CONCLUSIONS}

We performed specular and diffuse polarized neutron scattering of AF-coupled $\mathrm{Co} / \mathrm{Cu}$ multilayers. The magnetization is found to be distributed in regions with large and small vertically correlated domains. Conventional magnetometry (MOKE and SQUID) yields a smaller value of the saturation field than the value determined from off-specular polarized neutron scattering. Thus, PNR reveals the magnetic state in more detail. Smaller domains most likely at the top of the ML with a size of $0.5-1.5 \mu \mathrm{m}$ are saturated at a lower field of $\approx 500 \mathrm{Oe}$, whereas the larger domains $\left(\geqslant l_{\|} \approx 20 \mu \mathrm{m}\right)$ beneath remain $\mathrm{AF}$ coupled beyond apparent saturation at $\approx 1.5 \mathrm{kOe}$. The evolution of the domain size along the growth direction is not related to the evolution of structures with the same lateral size. However, the decreasing interface roughness leads to a higher degree of vertical correlation. An unexpected peak near the $\frac{1}{2}$-order Bragg position is reported. The explanation of its origin needs further investigations.

\footnotetext{
*Author to whom correspondence should be addressed. Electronic address: A.Paul@fz-juelich.de

${ }^{1}$ For details look into various contributions in Ultathin Magnetic Structures, edited by B. Heinrich and J. A. C. Bland (SpringerVerlag, Berlin, 1994), Vol. II.

${ }^{2}$ P. Grünberg, R. Schreiber, Y. Pang, M. B. Brodsky, and H. Sowers, Phys. Rev. Lett. 57, 2442 (1986).

${ }^{3}$ S. S. P. Parkin, N. More, and K. P. Roche, Phys. Rev. Lett. 64,
}

2304 (1990).

${ }^{4}$ M. N. Baibich, J. M. Broto, A. Fert, F. Nguyen van Dau, F. Petroff, P. Etienne, G. Creuzet, A. Friederich, and J. Chazelas, Phys. Rev. Lett. 61, 2472 (1988).

${ }^{5}$ G. Binasch, P. Grünberg, F. Saurenbach, and W. Zinn, Phys. Rev. B 39, 4828 (1989).

${ }^{6}$ E. E. Fullerton, D. M. Kelly, J. Guimpel, I. K. Schuller, and Y. Bruynseraede, Phys. Rev. Lett. 68, 859 (1992). 
${ }^{7}$ A. Paul, T. Damm, D. E. Bürgler, S. Stein, H. Kohlstedt, and P. Grünberg, J. Phys.: Condens. Matter 15, 2471 (2003).

${ }^{8}$ S. Langridge, J. Schmalian, C. H. Marrows, D. T. Dekadjevi, and B. J. Hickey, Phys. Rev. Lett. 85, 4964 (2000).

${ }^{9}$ H. Holloway and D. J. Kubinski, J. Appl. Phys. 83, 2705 (1998).

${ }^{10}$ A. Paul, E. Kentzinger, U. Rücker, D. E. Bürgler, and P. Grünberg, Physica B 356, 31 (2004).

${ }^{11}$ R. M. Osgood III, S. K. Sinha, J. W. Freeland, Y. U. Idzerda, and S. D. Bader, J. Magn. Magn. Mater. 199, 698 (1999).

${ }^{12}$ J. A. Borchers, J. A. Dura, J. Unguris, D. Tulchinsky, M. H. Kelley, C. F. Majkrzak, S. Y. Hsu, R. Loloee, W. P. Pratt, Jr., and J. Bass, Phys. Rev. Lett. 82, 2796 (1999).

${ }^{13}$ C. H. Marrows and B. J. Hickey, Phys. Rev. B 59, 463 (1999).

${ }^{14}$ U. Rücker, B. Alefeld, W. Bergs, E. Kentzinger, and Th. Brückel, Physica B 276-278, 95 (2000); U. Rücker, W. Bergs, B. Alefeld,
E. Kentzinger, and Th. Brückel, ibid. 297, 140 (2001); for information on HADAS see http://www.fz-juelich.de/iff/wns_hadas

${ }^{15}$ B. P. Toperverg, in Polarized Neutron Scattering, Forschungszentrum Jülich Matter and Materials, Vol. 12 (Forschungszentrum Jülich GmbH, Jülich, Germany, 2002), p. 247.

${ }^{16}$ J. F. Anker and G. P. Felcher, J. Magn. Magn. Mater. 200, 698 (1999).

${ }^{17}$ A. Paul, E. Kentzinger, U. Rücker, D. E. Bürgler, and P. Grünberg, Phys. Rev. B 70, 224410 (2004).

${ }^{18}$ E. Kentzinger, U. Rücker, and B. P. Toperverg, Physica B 335, 82 (2003).

${ }^{19}$ J. Weissmüller, A. Michels, J. G. Barker, A. Wiedenmann, U. Erb, and R. D. Shull, Phys. Rev. B 63, 214414 (2001).

${ }^{20}$ A. Schreyer, J. F. Anker, Th. Zeidler, H. Zabel, C. F. Majkrzak, M. Schäfer, and P. Grünberg, Europhys. Lett. 32, 595 (1995). 